Cinémas

Revue d'études cinématographiques

Journal of Film Studies

\title{
L'opérateur de vues animées : deus ex machina des premières salles de cinéma
}

\section{Timothy Barnard}

Volume 12, numéro 2, hiver 2002

Cinéma et cognition

URI : https://id.erudit.org/iderudit/024885ar

DOI : https://doi.org/10.7202/024885ar

Aller au sommaire du numéro

Éditeur(s)

Cinémas

ISSN

1181-6945 (imprimé)

1705-6500 (numérique)

Découvrir la revue

Citer cet article

Barnard, T. (2002). L’opérateur de vues animées : deus ex machina des premières salles de cinéma. Cinémas, 12(2), 161-184.

https://doi.org/10.7202/024885ar
Résumé de l'article

Cet article se penche sur l'activité des projectionnistes du début du cinéma (1905-1912) telle qu'elle se pratiquait en Angleterre, en France et aux États-Unis. Ce faisant, l'auteur dresse le portrait des mouvements de syndicalisation, des conditions de travail et des responsabilités professionnelles des projectionnistes, tout autant que de la législation qui encadrait leur activité. 


\title{
L'opérateur de vues animées: deus ex machina des premières salles de cinéma ${ }^{1}$
}

\section{Timothy Barnard}

Pour Martin Heath

\begin{abstract}
RÉSUMÉ
Cet arricle se penche sur l'activité des projectionnistes du début du cinéma (1905-1912) telle qu'elle se pratiquait en Angleterre, en France et aux États-Unis. Ce faisant, l'auteur dresse le portrait des mouvements de syndicalisation, des conditions de travail et des responsabilités professionnelles des projectionnistes, tout alıtant que de la législation qui encadrait leur activité.
\end{abstract}

\section{ABSTRACT}

This article recounts a brief history of the early film projectionist (c. 1905-1912) in England, France and the United States, taking up topics such as unionisation drives, working conditions, professional duties, legislation, etc.

Cet être invisible qui se dérobe aux regards dans les mystères de sa cabine, le deus ex machina de l'exploitation (Le Fraper, [1912], p. 150). 
[...] les scènes qu'il anime et qui portent à l'enthousiasme la salle ne lui font attribuer aucun mérite propre; jamais il n'est applaudi, jamais il n'est cité, et pourtant c'est à lui que nous devons de nous divertir en sécurité. Un bon opérateur projectionniste de cinéma, c'est un monsieur que l'on pourrait aussi bien encourager que celui qui prend la vue, en le citant par exemple sur l'affiche comme on y cite ses confrères, le décorateur, le régisseur, le chef d'orchestre, les solistes, etc. Le spécialiste peut être aussi artiste, déployer autant de goût, de savoir que ses camarades les exploitants auraient tout intérêt à encourager de la sorte ces modestes techniciens, il [sic] y gagneraient parce qu'on saurait que chez un tel tout est assuré de se bien passer et d'être présenté correctement par X opérateur (Ducom, 1924 , p. 150$)$.

«Une nouvelle race de dégénérés». C'est ainsi qu'en 1910, dans le journal corporatif Moving Picture World, un certain H. F. Hoffman qualifiait les opérateurs de vues animées. Ce même Hoffman raconte comment ces individus "sales et grossiers", ces «spécimens primitifs» qu'étaient les opérateursprojectionnistes, pouvaient être vus chaque matin aux bureaux de distribution des films, là où ils se procuraient la pellicule qu'ils projetteraient le jour même dans les nickelodeons. Voici le spectacle auquel on pouvait assister dans ces bureaux:

[...] éclats de rire immodérés et puérils, chahut, combats de lutte sur le linoléum humecté de jus de tabac, cris injuricux, consommation excessive de cigarettes, entaillage du mobilier à l'aide de couteaux de poche, martèlement, tapes dans le dos, ainsi qu'un charabia profane et de bruyantes instances pour la distribution immédiate des bobines ${ }^{2}$ (notre traduction). 
Cette description en forme de récrimination servait non seulement à jeter le discrédit sur la bataille syndicale des opérateurs, lesquels souhaitaient obtenir des salaires décents et une reconnaissance de leur travail, mais aussi à l'expression d'une xénophobie à peine dissimulée à leur égard. Hoffman va jusqu’à inventer des surnoms stéréotypés pour les protagonistes de son article: "Spaghetti ", "Muzzletoff", "Pork and Beans", et "Rhymo the Monk", ridiculisé pour son accent typique de nouvel immigrant. La xénophobie est aussi sensible sur le plan de l'encadrement législatif du métier. Au cours de cette même année, la ville de New York instaura une loi exigeant de tout opérateur qu'il soit d'origine américaine ou citoyen naturalisé, ce qui, selon Billboard, accula probablement la moitié des mille opérateurs de la ville au chômage ${ }^{3}$. La loi fut récusée par la suite, mais pas avant que plusieurs opérateurs n'aient perdu leur emploi et quitté la ville. Quant aux propriétaires de salles de cinéma américaines, encore intouchés par les lois, ils étaient eux aussi majoritairement immigrants. En 1908, les autorités new-yorkaises estimaient que plus de quatre-vingt-dix pour cent des cent quatre-vingts nickelodeons étaient dirigés par des "étrangers et des incompétents ${ }^{4}$.

Un employé du comptoir de distribution, à qui Hoffman demanda de témoigner des comportements qui faisaient de ces bureaux un endroit comme "nulle part ailleurs sur terre" entre 11 heures et midi, répondit ceci : "Ils sont cinglés, tout simplement cinglés. C'est le seul plaisir qu'ils ont. Tout le reste de la journée, ils sont enfermés dans leur cage brûlante, même le dimanche, et cela leur cause des troubles de la cafetière ${ }^{5}$." Sur ce dernier point, l'informateur de Hoffman avait tout à fait raison: durant l'ère des premières salles de cinéma (1905-1912), les conditions de travail des opérateurs de vues animées étaient effroyables. Ces conditions, et la chute des salaires durant cette période, menèrent à la formation des premiers syndicats de l'industrie du film, et ce, non pas sur les plateaux de tournage, mais dans les cabines de projection. C'est à partir de cet ancrage dans l'industrie cinématographique que la I.A.T.S.E. (connue entre 1915 et les années trente comme "The International Alliance of Theatrical and Stage Employes [sic] and Moving Picture Machine Operators of the United States and Canada») commença, dès la fin

L'opérateur de vues animées: deus ex machina des premières salles de cinéma 
des années dix, à s'organiser dans les studios d'Hollywood (Ross, 1941, p. 8). Cependant, les syndicats d'opérateurs n'étaient pas seulement préoccupés par les conditions de travail et le salaire de leurs membres; ils faisaient aussi pression sur le gouvernement, ils menaient des campagnes d'information sur la sécurité (autant celle de l'opérateur que celle du public) et tentaient d'introduire l'opérateur de vues animées au sein de l'industrie du film en tant qu'artisan spécialisé, et dont l'équipement et l'habileté étaient essentiels au processus de production cinématographique.

Alors qu'à l'époque des projections itinérantes, le dispositif de projection, visible et audible à l'arrière de la salle, constituait l'une des attractions pour le public, l'opérateur et sa machine commencèrent à se soustraire aux regards des foules avec l'implantation croissante des premières salles de cinéma. Mis à part de rares exceptions ${ }^{6}$, le projectionniste de l'époque était un homme (les femmes seront absentes de ce métier jusqu'à la Première Guerre mondiale en Angleterre) travaillant habituellement seul (excepté en Angleterre où les assistants étaient fréquents dans les grandes salles) pendant de longues heures, confiné à une étroite cabine tapissée d'amiante, mal ventilée et d'une chaleur accablante. Les plaintes concernant le manque d'eau courante, de toilettes ou d'un escabeau dans ces cabines étaient alors chose courante. Aujourd'hui, nous verrions ces cabines, dont l'histoire des dispositifs cinématographiques ne fait nulle mention, comme n'étant guère mieux que des cercueils. Un règlement municipal parisien stipulait qu'elles devaient avoir des dimensions d'au moins 1,6 par 1,36 mètre (Kress, p. 96). Cette loi fut pourtant perçue comme draconienne par les exploitants. On trouvait dans cet espace le projecteur, les outils et le matériel nécessaires, les bobines de film et l'opérateur lui-même. En Angleterre, les cabines de projection préfabriquées respectant les codes de sécurité municipaux étaient très populaires. Une publicité du Bioscope, datant de 1908, en présente deux formats: six pieds par quatre ou quatre par quatre ${ }^{7}$. Aux États-Unis, F. H. Richardson conseillait vivement, dans la première édition de son Motion Picture Handbook datée de 1910 , que la cabine mesure au moins «7 pieds carrés par 6 pieds du plancher au plafond", conscient toutefois que cette dernière mesure, la hauteur d'un homme debout, serait difficile à obtenir 
dans les nickelodeons où la cabine prenait souvent la forme d'un pigeonnier construit au-dessus du foyer des spectateurs (Richardson, 1910 , p. $\left.54^{8}\right)$. Pourtant, cet endroit s'avérera idéal pour la fonction que nous attribuerons à l'opérateur.

La direction des cinémas faisait habituellement très peu d'efforts pour faire de ces véritables fourneaux un environnement de travail décent. En 1910, Bioscope écrivait que les cabines étaient rarement ventilées vers l'extérieur, bien que dans la plupart des cas cela aurait pu se faire aisément ${ }^{9}$. À Paris, un règlement stipulait simplement que «[...] chaque fois que cela était possible, la ventilation devra être faite directement à l'extérieur ${ }^{10}$ ". Cette clause ne sera révisée qu'en 1927, rendant la ventilation vers l'extérieur obligatoire, tout en faisant passer la dimension minimum des cabines à 8 mètres cubes (Guimpel-Levitzky, 1939, p. 6-10). Les opérateurs protestaient contre ces conditions et contre l'existence même de ces cabines, se plaignant du fait que les autorités, alors qu'elles réglementaient la conception du projecteur et de la cabine pour limiter les risques d'incendies, négligeaient les conditions de travail et les qualifications du projectionniste. Que la plupart des feux commençaient lorsque la pellicule inflammable passait devant la lampe au carbone, ce n'était selon eux qu'un mythe. Au contraire, les incendies étaient dus beaucoup plus fréquemment à des circonstances excluant le mécanisme de projection. Les flammes prenaient naissance, par exemple, lorsque la pellicule jonchait éparse le sol, lorsque les opérateurs fumaient dans la cabine ou étaient inattentifs à la lampe. La cabine, il est vrai, servait bel et bien à protéger le public en contenant les flammes. Mais l'absence de toute clause légale assurant des pratiques sécuritaires et la méconnaissance par le public de l'état des lieux faisaient de la cabine l'endroit idéal pour un incendie et un cercueil en puissance pour l'opérateur. D'ailleurs, le journal corporatif français Ciné-Journal décrivait, en 1911, la mort d'un opérateur n'ayant que quatre jours d'expérience dans son domaine: "[Antoine] Schmidt devait pénétrer dans la cabine par un trou pratiqué dans le plancher et qu'on atteint au moyen d'une échelle. Une fois à l'intérieur, l'opérateur refermait l'ouverture. C'était une véritable souricière ${ }^{11}$." Pour un opérateur américain, écrivant au Moving Picture World en 1908, la cabine de projection était à la fois inu- 
tile pour la protection du public et dangereuse pour la santé de l'opérateur:

De nos jours, un opérateur doit se procurer une licence après avoir passé un examen rigide [cette pratique était loin d'être universelle à cette date]. Il est alors considéré comme étant capable de manipuler correctement sa machine. Cette dernière, après avoir été équipée avec toutes les pièces connues pour protéger la pellicule, est inspectée et passée en revue par les assureurs et déclarée à l'épreuve du feu par le capitaine des pompiers.

Quelle est alors l'utilité de cette cabine? S'il s'agit de porter atteinte à la santé de l'opérateur, elle remplit assurément sa mission.

Laissez l'opérateur se nettoyer les narines avec un mouchoir propre après une dure journée de travail. Noir, n'est-ce pas? [...] Laissez-le examiner les traces de poussière de carbone sur le dessus de la lampe, de la tablette qui soutient le projecteur, etc., après avoir opéré sa machine. Il y en a partout, n'est-ce pas?

L'opérateur respire constamment cette poussière... Elle est hautement nocive pour les poumons, la gorge et les membranes du nez. De cette irritation peuvent résulter pneumonie, pleurésie, amygdalite et cataractes chroniques du nez ${ }^{12}$ (notre traduction).

De plus, selon cet opérateur américain, le projectionniste n'était pas un travailleur manuel incompétent, comme les dirigeants le prétendaient afin de provoquer la chute des salaires, mais plutôt, tel que Jacques Ducom le suggérait dans l'épigraphe de cet article, un artisan qualifié: «Aussi longtemps que la loi obligera l'opérateur à travailler dans sa cabine, ce dernier restera le plus misérablement payé dans les rangs des amuseurs professionnels ${ }^{13} . »$

Les tâches de l'opérateur dans les toutes premières salles de cinéma étaient nombreuses. (Pour les autres événements qui avaient lieu à cette période et dans lesquels les films n'étaient qu’un divertissement secondaire, comme dans les spectacles de music-hall anglais, les tâches de l'opérateur étaient plus circonscrites). Comme le décrivait le Nickelodeon en 1909, les tâches du projectionniste induaient non seulement le fonctionnement du projecteur, mais le réglage des lumières de la salle et des haut- 
parleurs du trottoir, la direction des musiciens, la projection des diapositives accompagnant les chansons et, entre les films, le rembobinage et la réparation de la pellicule ${ }^{14}$. À cela, nous pourrions ajouter, par exemple, la réparation et le bon fonctionnement de projecteurs non standards et mal conçus, et l'emploi de technologies pour le moins expérimentales et sans doute fort capricieuses, comme ces dispositifs de son synchrone introduits en 1907: le Chronophone de Gaumont, le Vivaphone de Hepworth ainsi qu'une poignée d'autres systèmes encore moins connus. Un autre commentateur écrivait dans Nickelodeon, peu de temps après: "[...] l'opérateur est plus ou moins responsable de toute la salle ${ }^{15}$.». De son côté, Ducom comparait l'opérateur à un pilote d'avion à cause de la quantité de tâches qu'il devait remplir très rapidement avec seulement deux mains (Ducom, 1924, p. 445).

S'ajoutaient à cela deux corvées, qui ne sauraient être oubliées du moment qu'un film était projeté: surveiller la lampe à arc (pour assurer la bonne qualité des images et pour prévenir les incendies) et tourner la manivelle pour amener la pellicule dans le projecteur. Le scintillement de l'image était un problème constant à cette période et seuls un bon faisceau de lumière et une rotation précise de la manivelle pouvaient le minimiser. L'opérateur de "première classe " pouvait même, disait-on, varier la vitesse de projection afin d'obtenir ou d'augmenter certains effets désirés dans des séquences précises d'un film. Mais première classe ou non, aucun opérateur-projectionniste ne pouvait éviter l'effort et la monotonie implicites au roulement de la manivelle. Selon la machine, soixante-dix à cent vingt tours de manivelle étaient nécessaires pour chaque minute de film projeté, et ce, afin d'obtenir la vitesse minimum de seize images par seconde qui était la plupart du temps dépassée. Après une journée de travail de dix à douze heures, on raconte que les opérateurs répétaient convulsivement ce mouvement circulaire du bras durant leur sommeil (Musser, 1990, p. 441).

Malgré l'importance cruciale des opérateurs pour les premières salles de cinéma, l'ignorance des cours du marché par la plupart des propriétaires de cinémas et l'augmentation explosive du nombre de salles ${ }^{16}$, on remarque à cette période une chute de leurs salaires et une détérioration de leurs conditions de travail. Ce paradoxe est surtout apparent aux États-Unis, qui connaissent à la 
fois la hausse la plus spectaculaire du nombre de salles de cinéma et les attaques les plus virulentes concernant le statut et les revenus des opérateurs. En 1909, le Moving Picture World offrait une explication commode de ce phénomène. Il suggérait que les salaires élevés attribués aux opérateurs dès l'apparition des salles de cinéma avaient attiré des foules de travailleurs inexpérimentés, prêts à apprendre en cours de travail mais pour un salaire moindre, et qui, ce faisant, avaient fait dégringoler par inadvertance les salaires élevés auxquels ils aspiraient ${ }^{17}$. Dix mois auparavant, le même journal corporatif, qui offrait aux opérateurs et propriétaires de cinéma un service gratuit d'embauche de main-d'œuvre, mentionnait qu'il ne pouvait plus placer les opérateurs, alors qu'il n'arrivait pas à satisfaire la demande six mois plus tôt ${ }^{18}$. Ce flot de simples "pousseurs de manivelle" dans les rangs des opérateurs contribua non seulement à faire chuter les salaires, mais à diminuer la qualité des représentations et à rendre primordiale la question des incendies pour les premières salles de cinéma.

En outre, il est possible d'aborder cette campagne contre l'opérateur d'un point de vue idéologique. De toute évidence, les propriétaires ne voulaient pas reconnaître à l'opérateur ses compétences ni son importance dans la salle de cinéma. Aux alentours de 1906-1910, avant même la construction standardisée de systèmes de projection motorisés, avant même l'avènement du long métrage et l'abandon du spectacle de variétés, les directeurs voulaient que la projection soit une tâche mécanique non spécialisée, avec la situation et le salaire que cela suppose, alors que ce n'était évidemment pas le cas. Cet exemple tiré de Views and Film Index (appelé plus tard Film Index), qui appartenait aux maisons de production Vitagraph et Pathé, résume bien la position des exploitants à la suite des agitations ouvrières de 1908 :

\footnotetext{
Qu'est-ce qu'un opérateur de vues animées? Un employé qui a certaines fonctions. Rien de plus, rien de moins. Le fait qu'il trimbale dans sa poche une licence accordée par une quelconque ville ne change en rien la situation... Il est un employé et c'est ainsi qu'il doit considérer et garder sa place.

Ces déclarations sont une réponse aux nombreux rapports envoyés par des exploitants, qui ont beaucoup de
} 
problèmes avec ces opérateurs qui croient que la position attribuée par leur licence leur confere plus de privilèges qu'à ceux qui ont investi du capital dans le spectacle $^{19}$ (notre traduction).

Malgré son mépris envers les travailleurs, l'auteur de cette diatribe est obligé de reconnaître l'exploitation dont les opérateurs sont victimes:

En parlant de ce sujet, voici un conseil pour l'exploitant concernant ses propres actions. L'opérateur est un être humain, pas un moteur électrique ou autre appareil mécanique. Il doit manger, se reposer et dormir. $\mathrm{Ne}$ privez pas l'opérateur de ses repas ou du repos nécessaire. Mettez-vous à la place de l'opérateur (ou dans sa cabine) et agissez en conséquence ${ }^{20}$ (notre traduction).

Quel était exactement le salaire d'un opérateur à cette époque? En Angleterre, plusieurs situations et conditions de travail coexistaient. Certains opérateurs étaient réellement de simples "pousseurs de manivelle", deux heures par soir dans un musichall; l'entretien de l'appareil et de la pellicule était de la responsabilité de l'électricien rattaché à la salle, lequel remplissait parfois la tâche d'opérateur durant la soirée pour arrondir ses fins de mois. Bioscope déclarait, tôt en 1909, que le salaire d'un opérateur à temps plein en Angleterre variait entre 1 livre 5 shillings et 3 livres 10 shillings $^{21}$. Plus tard la même année, un opérateur se plaignait au Bioscope que bien qu'il considérait qu'un revenu de 3 livres pour une semaine normale de travail était honnête, un salaire maximum de 2 livres était envisageable, et ce, uniquement en doublant leurs quarts de travail, pour en arriver à 1415 heures par jour ${ }^{22}$. En 1911-1912, remarque Rachael Low, le salaire moyen était d'environ 25-30 shillings par semaine (Low, 1949, p. 70); en 1910 Bioscope estimait que 30 shillings était un salaire convenable, le comparant favorablement aux 35-42 shillings que gagnaient les employés spécialisés comme les plombiers et les électriciens, reconnus pour leurs heures de travail bien plus longues ${ }^{23}$. En France, les opérateurs syndiqués gagnaient 60 à 70 francs par semaine en 1910, alors que les non-syndiqués recevaient 40 à 45 francs $^{24}$. Au début de 1911, le 
syndicat français des opérateurs de vues animées promettait de faire cesser la chute des salaires et d'exiger un revenu quotidien minimum de 10 francs ${ }^{25}$.

La situation aux États-Unis était encore plus grave. C'était chose courante que d'avoir des projections continues depuis le début de l'après-midi jusque tard le soir. Pour assurer ces projections, un petit cinéma n'engageait qu'un seul opérateur, lequel était relayé, le temps de courtes pauses, par le propriétaire ou un autre employé. En 1908, un opérateur expérimenté demandait 25 à 30 dollars par semaine. Un an plus tard, précisément après que le nombre de salles de cinéma ait augmenté de façon inouïe au pays, des opérateurs protestaient contre des offres allant aussi bas que 14 dollars par semaine $^{26}$. On rapporte même en Georgie, plus tôt cette année-là, un revenu de 10 dollars par semaine ${ }^{27}$. Il semble que tout le monde voulait sa part du gâteau : déjà en 1907, quelqu'un se plaignait dans Moving Picture World qu'à Cincinnati, deux délégués syndicaux de leur profession respective, un charpentier et un poseur de canalisations, s'étaient ouvert un nickelodeon et payaient les opérateurs 9 à 10 dollars par semaine, soit beaucoup moins que les 25 à 40 dollars exigés par les professionnels de l'époque:

Ces hommes sont des champions du travail syndical. Ils reçoivent un salaire de leurs syndicats pour procurer à leurs hommes des revenus d'ordres syndicaux, mais dès qu'ils deviennent employeurs à leur tour, leurs nobles principes disparaissent au profit des désirs les plus sordides de la nature humaine ${ }^{28}$ (notre traduction).

En 1911, alors que David Hulfish publiait un exemple de budget à l'intention des gens aspirant au rang d'entrepreneurs, un revenu hebdomadaire de 18 dollars était considéré adéquat pour un projectionniste "première classe" (contre $18 \$$ pour le chanteur, $15 \$$ pour le pianiste et $5 \$$ pour le portier et le caissier - Hulfish, 1911, p. 183). Il est intéressant de noter que les reçus hebdomadaires de ce budget idéal totalisent 340 dollars de revenus contre 214,50 dollars de dépenses. Il s'agit d'un profit généreux compte tenu de l'investissement minimum requis par celui qui s'improvise directeur de salle de cinéma et qui n'a probablement aucune expérience ni expertise dans le domaine. 
Ce profit ne justifie surtout pas qu'on économise quelques dollars aux dépens de l'opérateur, et ce, au détriment de la qualité de la représentation et de la sécurité de l'audience. En novembre de la même année, les opérateurs de Baltimore font la grève pour l'obtention d'une échelle salariale décente: $20 \$$ pour dix, $22 \$$ pour onze et $24 \$$ pour douze heures de travail avec une pause d'une heure (un quart de travail de moins de douze heures, semble-t-il, ne méritait pas de pause d'une heure); et $24 \$$ pour 13 heures avec deux pauses d'une heure ${ }^{29}$. Faire la grève afin d'avoir $24 \$$ pour pousser une manivelle soixante-dix-huit heures par semaine dans une cabine bouillante, exiguë et mal ventilée, et ne pas être payé de la sorte par quelqu'un qui n'a aucun savoir ou talent particuliers, mais qui fait $125 \$$ par semaine, selon l'exemple de Hulfish, sur un investissement d'à peine $2000 \$$ ! Mais l'octroi de revenus équitables était impossible à obtenir dans le milieu non syndiqué des premières salles de cinéma. Dans sa couverture de la grève, Billboard fait remarquer avec satisfaction que jusqu' ici "[...] aucune salle ne semble avoir souffert, les places de ceux qui quittent étant rapidement comblées ${ }^{30}$ \%.

En septembre 1908, les opérateurs de Chicago firent la grève pour des salaires de 22,50\$ par opérateur s'occupant d'une seule machine et de $20 \$$ chacun pour deux opérateurs s'occupant de deux projecteurs (une nouveauté à l'époque). La réformatrice sociale Jane Addams estimait que le sixième de la population de Chicago, devenu le plus grand marché de spectacles animés, allait se masser tous les dimanches soirs dans ses quelque quatre cent soixante-six salles ${ }^{31}$. Des salles que menaçaient d'ailleurs de fermer les opérateurs si elles ne respectaient pas les tarifs syndicaux. Les chanteurs de chansons illustrées se joignirent à eux, en se donnant gratuitement en spectacle à l'extérieur des salles, tout en encourageant les gens à ne pas entrer ${ }^{32}$. Le tout nouveau syndicat de la ville (Nickel Theatres Operators' Union) présentait des diapositives avant les chansons illustrées et les films, assurant le public que " $[\ldots]$ les employés de la place faisaient partie du syndicat des acteurs [I.A.T.S.E.] et que la salle opérait selon les "règles" de l'art ${ }^{33}$ ". En 1917, le I.A.T.S.E. adoptait un insigne, lequel était affiché au comptoir de vente afin d'informer le public de la syndicalisation ou non de l'opérateur (Baker, 1933 ${ }^{34}$ ). 
Si l'offensive des exploitants menée contre l'amélioration de la situation et des salaires des opérateurs était autant idéologique que pratique, son but n'étant pas uniquement de réduire les dépenses mais de soutenir les droits du capital, selon l'explication succincte du Film Index, c'était aussi le cas pour la campagne syndicale des opérateurs. Les syndicats naissants en France, en Angleterre et aux États-Unis ont tous imaginé des stratégies remarquablement semblables, à des degrés de succès différents, au moment de négocier avec les dirigeants, mais aussi au-delà de ces négociations, en faisant appel au public, aux autorités gouvernementales et aux autres secteurs de l'industrie cinématographique. Dans tous les cas, la bataille a été menée au nom du principe suivant : que l'opérateur était un artisan spécialisé et un participant essentiel à la production filmique.

Les premiers appels des opérateurs adressés à leurs dirigeants adoptaient un ton conciliant et corporatiste de partenariat entre le capital et la main-d'œuvre spécialisée. Dans la plupart des cas, les travailleurs concernés étaient des opérateurs expérimentés, issus de l'industrie du film telle qu'elle avait été à ses débuts, et donc très peu habitués à ce qu'on les traite comme des ouvriers. Le plan était simple: en payant quelques dollars de plus, la direction pouvait s'assurer d'avoir des projections professionnelles, c'est-à-dire des opérateurs fiables et ponctuels qui ne fumaient ni ne buvaient au travail, une maintenance adéquate de la pellicule et de l'équipement, des présentations de haute qualité, remplies d'effets dont raffolait l'assistance, exemptes de scintillements, de ruptures de la bande, et bien sûr d'incendies. Un bon opérateur rapporterait plusieurs fois son salaire en clientèle ravie, comme le suggérait Ducom. Le corollaire qui s'ensuivait, comme le faisait remarquer Jean Mariani, chef du syndicat français, est que "le bon marché coûte toujours fort cher ${ }^{35}$ ".

Il y avait quelques rapports encourageants de la part des syndicats d'opérateurs indiquant que cette proposition commençait à porter fruit. En 1908, le syndicat de San Francisco, probablement le tout premier syndicat d'opérateurs à être formé (il débuta en 1904 comme une "association protectrice » indépendante et, après 1907, devint une section syndicale du I.A.T.S.E.), affirmait que les exploitants étaient "[...] prêts à payer selon le 
barème de salaires [...] afin d'obtenir des hommes fiables ${ }^{36} \%$. Mais, pour la plupart, les exploitants ne respectaient aucun barème. Dans tous les cas, comme l'indique Michael Chanan en parlant de l'opérateur britannique, "l'audience venait de toute façon" (Chanan, 1976, p. 41), quelle que soit la qualité du spectacle. Les exploitants faisaient un joli profit en offrant des spectacles de moindre qualité et n'avaient pas besoin de maind'œuvre syndiquée pour leur enseigner la façon de diriger leurs affaires. D'ailleurs, les exploitants n'appréciaient guère l'idée qu'un statut particulier soit attribué à l'opérateur, qui faisait de lui le seul employé spécialisé des premières salles de cinéma. La prolétarisation de l'opérateur était essentielle à l'essor du secteur de l'exploitation et au développement d'un produit de consommation culturel standardisé pouvant être présenté par à peu près n'importe qui, si on relègue au second plan les questions d'entretien, de qualité et de sécurité. On en est venu à appeler ce produit le cinéma classique, et ce n'est pas un hasard si sa codification coïncide avec la transformation du statut de l'opérateur, de celui d'artiste à celui de prolétaire.

Jetés dans une impasse par les exploitants, les opérateurs firent appel aux producteurs et aux distributeurs dans leur campagne pour une reconnaissance de leur position vitale au sein de la production filmique. Cette stratégie était en quelque sorte condamnée d'avance par l'intégration verticale de l'industrie cinématographique. Pathé, par exemple, était à la fois le plus gros producteur et le plus important exploitant de France. Dans pareil cas, on imagine difficilement de sa part une réponse sympathique aux revendications des opérateurs, surtout lorsque ces revendications sont explicitement liées à la syndicalisation et à la standardisation des salaires. Cela n'a pas empêché le syndicat des opérateurs français, les Opérateurs cinématographistes de France, fondé en 1908, de mettre de l'avant une rhétorique de coopération dans les différents secteurs de l'industrie, qui était hautement corporatiste. Ce syndicat envisageait une industrie du film composée de quatre partenaires: producteurs, distributeurs, exploitanits et opérateurs furent tous invités à la même table en 1908 afin de débattre la représentation des films ${ }^{37}$. Tout porte à croire que les opérateurs n'étaient pas pris au 
sérieux: le capital, et non pas la main-d'œuvre, s'assurait de trouver tous les associés nécessaires à l'industrie.

Une rhétorique corporatiste du même genre émergea aux États-Unis. Les opérateurs firent appel aux producteurs (Motion Picture Patents Company) et aux distributeurs (Film Service Association) afin que ceux-ci s'opposent aux mauvais maniements de leurs films et à leurs piètres représentations à cause d'opérateurs non qualifiés ${ }^{38}$. Pourtant, il n'existe aucun document suggérant une quelconque réponse, voire le moindre intérêt pour cette mobilisation, sinon de la part des opérateurs et de quelques chroniqueurs de la presse spécialisée. Bien qu’un personnage aussi important que Charles Urban en Angleterre affirmait, comme le rapporte la lettre d'un opérateur au rédacteur de Bioscope, que les feux étaient causés par l'opérateur et non uniquement par un équipement défectueux ${ }^{39}$ - ce qui était précisément la position des syndicats, puisqu'ils tentaient d'expulser de la profession les "pousseurs de manivelles» sous-payés et inexpérimentés - , cela ne suggère en rien qu'Urban encourageait la campagne de reconnaissance des opérateurs.

Après l'échec de cette stratégie, la campagne des opérateurs se concentra sur la question de la réglementation gouvernementale et de l'émission de permis. Les syndicats d'opérateurs des trois pays manifestèrent avec acharnement pour l'obtention d'une réponse formelle à cette question, arguant qu'en plus de la qualité du spectacle, la sécurité du public était en cause. De plus, l'octroi de permis protégerait les opérateurs inexpérimentés contre eux-mêmes : en 1911, un garçon de 16 ans s'était électrocuté dans sa cabine en Virginie ${ }^{40}$. En 1909, un Marseillais avait laissé son emploi de plongeur pour le boulot de projectionniste, au salaire plus alléchant, malgré le fait que ce dernier eût chuté de 10 à 3 francs par jour. Trois jours plus tard, il mettait le feu à sa cabine et provoquait une débandade dans la salle ${ }^{41}$. Deux semaines plus tôt, le syndicat notait avec indignation deux autre feux ${ }^{42}$ juste après que le préfet de police de Paris eût ignoré les requêtes des opérateurs qui exigeaient qu'on ajoute aux règlements municipaux l'obtention obligatoire du permis de projection. Le syndicat se plaignit amèrement de ne même pas avoir été reçu devant la Commission technique chargée d'élaborer le 
règlement et d'entendre les recommandations des divers secteurs de l'industrie ${ }^{43}$. Même quelques exploitants ne pouvaient nier les bénéfices d'une telle réglementation pour les opérateurs. Aussi tard qu'en 1911, à la suite d'un autre incendie ayant pris naissance dans la cabine d'un cinéma itinérant où œuvrait un novice, un exploitant se posait la question: "Quand exigerat-on des opérateurs qu'ils soient munis d'un brevet de capacité, comme le chauffeur d'auto ${ }^{44}$ ?» Pourtant, le gouvernement n'agissait toujours pas, même si, par ailleurs, il exigeait des propriétaires de grandes salles la présence d'un médecin sur place en tout temps. Ainsi, le syndicat était responsable de certifier ses propres membres - sur la base des expériences de travail et des références et non, comme en Angleterre, à partir d'un examen pratique - avant de se dissoudre un peu plus tard la même année, incapable d'améliorer l'échelle salariale ou d'obtenir une réglementation satisfaisante.

Une histoire semblable impliquant un gouvernement inactif et un syndicat impuissant s'est déroulée en Angleterre, où la National Association of Cinematograph Operators (N.A.C.O., affiliée à la National Association of Theatrical Employees, N.A.T.E.) avait été fondée en 1907. Dès ses débuts, la N.A.C.O. faisait pression sur le London County Council (L.C.C.) pour inclure dans le règlement sur la projection des films la tenue d'examens en vue de l'octroi de permis aux opérateurs. À plusieurs occasions, en 1907 et en 1908, le syndicat et un journal spécialisé partisan de la réglementation annonçaient avec espoir qu'ils arriveraient à persuader le L.C.C. d'évaluer les opérateurs ou de reconnaitre les examens de certification du syndicat ${ }^{45}$. Mais le L.C.C. a rapidement déçu ces attentes. Son expert technique déclarait en février 1908 que seul un projet de loi national pouvait se charger de tels examen ${ }^{46}$. Mais lorsqu'une loi nationale apparut plus tard la même année (Cinematograph Act), elle resta silencieuse sur tout ce qui concernait l'opérateur ${ }^{47}$. Une réglementation existait bel et bien à la grandeur du pays en ce qui avait trait à la cabine et au projecteur, mais elle fermait les yeux sur le problème des opérateurs non qualifiés. Les pressions des exploitants contre la certification des opérateurs, perçue comme la rampe de lancement de la syndica- 
lisation, étaient elles aussi vigoureuses: «la syndicalisation par loi parlementaire" incarna la riposte des exploitants contre ces appels des syndicats à la certification des opérateurs (Low, 1949, p. 67). En France, le syndicat n'hésita pas à dire qu'il s'agissait là de son but ${ }^{48}$.

Dépourvu de l'outil organisationnel qu'aurait représenté l'authentification des opérateurs, la N.A.C.O. a dépéri jusqu'à ne devenir qu'un petit syndicat inefficace. En avril 1910, elle avouait n'avoir plus que deux cents membres et non pas les deux mille espérés ${ }^{49}$. Rachael Low affirme que l'année suivante connut une hausse du recrutement et que le syndicat atteignit mille membres en 1911, mais elle fait remarquer qu'il ne s'agissait que d'une fraction du nombre total d'opérateurs dans le pays, où il y avait au moins quatre mille salles de cinéma officielles (Low, 1949 , p. 70). Cette année-là, alors que la N.A.C.O. émettait un autre appel à la certification obligatoire, Bioscope se plaignit qu’à cet égard l'industrie du film britannique traînait « loin derrière les États-Unis ${ }^{50}$ ".

C'est en fait aux États-Unis que, assez étonnamment d'ailleurs, les lobbies des opérateurs eurent le plus de succès, bien que la bataille fut menée à l'échelle municipale ou des États, et non pas à celle de la nation. La certification généralisée ne s'est pas nécessairement traduite, toutefois, en cette croissance du syndicat et de son pouvoir de négociations tant espérés. Jusqu'en juin 1910, des sections syndicales du I.A.T.S.E. existaient dans seulement dix-huit villes des États-Unis et du Canada ${ }^{51}$, et bien qu'aucun chiffre précis ne soit disponible, le total des membres de ces sections devait se dénombrer bien en deçà des dix à quinze milles opérateurs employés à cette époque.

Initialement, les autorités américaines, comme celles d'Europe, étaient satisfaites de pouvoir mieux encadrer les cinémas, d'inspecter les projecteurs et de réglementer la construction des cabines de projection. Certaines municipalités obligèrent même les propriétaires de salles à engager à leurs frais un pompier municipal pour surveiller les représentations. Mais aussi tôt qu'en 1907 , des États comme le Massachusetts et l'Illinois entreprenaient de délivrer des certificats aux opérateurs. New York suivit au début de 1908 , date à laquelle on retrouve des chartes de certification 
des opérateurs dans trente-trois États ${ }^{52}$. Les opérateurs se plaignaient (!), toutefois, que l'examen n'était pas suffisamment rigoureux ou technique et que certains évaluateurs étaient incompétents. Dans tous les cas, malgré des appels passionnés pour un syndicat d'envergure nationale ${ }^{53}$, la syndicalisation des opérateurs resta impossible dans un pays aussi grand que les États-Unis et au sein d'une industrie dont la main-d'œuvre, à cette époque, était très dispersée.

Michael Chanan (1976, p. 15 sq.) raconte qu'en Angleterre, l'échec des operateurs dans leur tentative de s'organiser sur une grande échelle fut causé par leur adhésion au mauvais type de syndicat : à un syndicat «de métier» démodé plutôt qu’à un syndicat moderne de type "industriel». Chanan affirme que les opérateurs auraient eu beaucoup plus de succès dans leur tentative de syndicalisation s'ils avaient mené leur campagne en mettant de l'avant une syndicalisation commune à tous les ouvriers, spécialisés ou non, œuvrant dans des cinémas de propriété individuelle. Il cite d'ailleurs un exemple de ce genre où, à l'aube de la guerre, tout le monde, jusqu'au vendeur de chocolat, était syndiqué. Selon Chanan, les propriétaires de cinémas privés étaient vulnérables aux menaces de fermeture et désiraient en finir avec le syndicat afin que les affaires reprennent; la syndicalisation d'un ou de deux opérateurs dans chacun des milliers de cinémas, mentionne-t-il, était un cauchemar logistique.

Il est difficile de défendre les mérites d'une stratégie qui n’a eu qu'un succès limité, plutôt que celle que préconise Chanan et qui n'a jamais été mise à l'épreuve, mais c'est ce que j'essaierai de faire ici. Chanan a certainement raison à propos du défi organisationnel soulevé par la stratégie des syndicats "de métier ", mais rien ne prouve que l'option de rechange qu'il propose aurait été plus efficace à long terme. Les travailleurs non spécialisés sont difficiles à organiser - ils viennent et puis s'en vont, sont facilement remplaçables et n'ont souvent pas développé la conscience qui les pousserait à se joindre à un syndicat. De plus, dans les lieux de travail où s'emploie surtout de la main-d'œuvre non spécialisée, on tente par tous les moyens de démanteler les syndicats. Et quand les travailleurs parviennent à s'organiser, on constate rarement un quelconque progrès sur le plan des réclamations 
salariales, précisément parce que ces travailleurs ne sont pas spécialisés. L'exemple de Chanan, datant de 1914, est à ce titre très éloquent. De plus, il est difficile de comprendre comment une telle stratégie favoriserait une propagation des syndicats dans l'industrie (les petites entreprises syndiquées une à une ont peu de chances "d'infecter» des établissements similaires). Et puis quel serait le pouvoir de négociation de l'opérateur et quel serait son succès, s'il ramenait son propre statut à celui de simple travailleur industriel, ce qui était d'ailleurs la position des exploitants qui rêvaient de faire passer leur salaire de celui d' "artisan" à celui d'ouvrier?

Les syndicats d'opérateurs, de type "artisan », dits démodés, s'imposaient malgré tout dans plusieurs pays. Encore une fois, les États-Unis ont ouvert la voie, malgré une tradition de militantisme ouvrier et syndical moins grande qu'en Europe, et des défis logistiques de plus grande envergure. Le nombre de salles, leur dispersion géographique et l'expansion fantastique de l'industrie créaient en fait un réel besoin pour des opérateurs non spécialisés puisque, de toute façon, le nombre d'opérateurs spécialisés ne répondait pas à la demande. La stratégie des opérateurs était alors de s'organiser une ville à la fois, d'appeler au boycott des cinémas non syndiqués où il y avait des projectionnistes spécialisés et où le syndicat avait une chance de l'emporter sur le propriétaire. Le syndicat de San Francisco reportait le succès de cette tactique ${ }^{54}$ dans l'espoir qu'une fois atteint une certaine masse critique, les cinémas de propriété individuelle se mettraient à tomber. Dans une certaine limite, c'est ce qui se produisit, aux États-Unis du moins. Nous retrouvons là la tactique avancée par Chanan, celle de mettre à l'épreuve la vulnérabilité des propriétaires de cinémas individuels. Mais dans ce casci, la tactique fut orientée vers le pivot de l'opération: le projectionniste spécialisé.

Toutefois, Chanan a peut-être raison sur un point, bien que ce ne soit pas au sens où il l'entendait, lorsqu'il dit que les opérateurs anglais, beaucoup moins favorisés par le succès, étaient dans le "mauvais syndicat». Mauvais parce que le N.A.C.O. n'était simplement pas un syndicat très militant ou efficace, tel que Chanan lui-même l'a démontré en détail. Mais cela ne 
mène pas nécessairement à la conclusion que la stratégie du syndicat de type "artisan" était une erreur. Cela suggère toutefois une question intéressante, mise en lumière par les violents conflits d'attribution entre les syndicats américains, et plus particulièrement entre les syndicats anglais. Dans les deux cas, les syndicats industriels et théâtraux se disputaient l'allégeance des opérateurs ${ }^{55}$. Qui était, au fait, un opérateur? Un travailleur industriel à demi spécialisé ou un artiste de la scène des temps modernes? Personne n'arrivait à s'entendre là-dessus. En France, peu après la guerre, les exploitants et les compagnies d'électricité ne pouvaient s'entendre sur la tarification de l'électricité dans les cinémas: le projecteur était-il une machine industrielle ou une source de lumière (Rosenblatt, 1998, p. 47-49)?

Les opérateurs des trois pays ont imaginé une stratégie commune et très visible pour vaincre les obstacles à une action collective exigée par leur profession, et ce, en faisant appel à la presse spécialisée émergente, où ils furent chaudement accueillis. En Angleterre, le journal Kinematograph and Lantern Weekly s'offrait, dès sa première parution, comme l'organe officiel du syndicat naissant ${ }^{56}$ et promettait de fournir une représentation active et partisane des intérêts des opérateurs. La plus grande revue professionnelle en Angleterre, Bioscope, fondée en 1908, faisait un relevé plus limité des activités des opérateurs, mais approuvait leur certification ${ }^{57}$ et garantissait une couverture complète des activités syndicales, des écoles de formation et des autres questions d'ordre technique, ainsi que la publication des lettres ouvertes des opérateurs. À un certain moment, le syndicat proposait comme avantage à ses membres un abonnement gratuit à la revue, payée par le syndicat, apparemment en échange de la publication des informations d'ordre syndical ${ }^{58}$. Bien qu'en 1910 Bioscope eût édité le Modern Bioscope Operator ${ }^{59}$, parmi la vingtaine de manuels techniques publiés avant la guerre dans les trois pays, il devenait la même année l'organe officiel de la Cinematographic Defence League, un groupe de pression composé d'exploitants de salles de cinéma. Durant cette période, le journal est devenu de moins en moins réceptif aux griefs des opérateurs et particulièrement à leurs demandes salariales ${ }^{60}$. 
La plus importante revue professionnelle en France, CinéJournal, lancée en 1908, s'opposait dans ses éditoriaux au préjugé "antisyndical " ressenti chez les opérateurs eux-mêmes ${ }^{61}$ et ouvrait ses colonnes aux chefs syndicaux pour faire le point sur la certification, la standardisation des salaires et les différents griefs. Quand le syndicat disparut en 1911, étant incapable d'imposer un salaire fixe ou d'instaurer une amélioration des conditions de travail et de sécurité au sein de l'industrie - précisément parce que ce n'était pas un syndicat en soi mais une association volontaire sans réel pouvoir de pression sur les exploitants -, un nouveau journal publié par Charles Le Fraper, Le Courrier cinématographique, prit la relève. Le Fraper offrait de payer tous les frais de L'Amicale des opérateurs et cinématographistes (Jean Mariani, le défunt chef du syndicat, avait prédit la résurrection du groupe en bannissant le mot syndicat et en supprimant les cotisations), et fournissait même de l'espace dans ses bureaux. Reconnaissant la difficulté de réunir les opérateurs pour discuter de leurs problèmes communs, il ajoutait: «Les communications seront faites dans les colonnes du Courrier et nous proposons aujourd'hui de déléguer à un de nos rédacteurs, la lourde tâche d'en centraliser les forces ${ }^{62}$."

La toute première parution de ce qui allait devenir la plus importante revue professionnelle de l'époque aux États-Unis, le Moving Picture World ${ }^{63}$, contenait des lettres ouvertes d'opérateurs activistes réclamant un salaire convenable pour les opérateurs et la formation d'une ligue pour les représenter. L'éditeur mentionnait à la suite de ces lettres: « Nous sommes bien disposés à faire du Moving Picture World l'organe officiel de la ligue et de rapporter son développement de temps à autres. » L'entête de la revue l'identifiait comme «le seul journal hebdomadaire indépendant publiant les intérêts des fabricants et des opérateurs", reflétant ainsi le désir des opérateurs d'être acceptés au sein de l'industrie. Durant l'ère du nickelodeon, le journal offrait un service d'annonces gratuites pour les opérateurs cherchant du travail, publiait les lettres ouvertes des opérateurs, se positionnait en faveur de la certification, de la syndicalisation et de l'amélioration des salaires et des conditions de travail, et publiait régulièrement une chronique technique plutôt engagée, écrite par 
F. H. Richardson, le plus bruyant et influent défenseur des opérateurs dans l'industrie.

Moving Picture World affirmait aussi son pouvoir unique de rassembler les opérateurs dispersés. Dans l'un des premiers éditoriaux on notait: "Nous aimerions tenir une réunion de ceux qui sont intéressés [...] mais puisque les opérateurs sont trop loin les uns des autres pour qu'une telle réunion ait lieu, nous devons forcément avoir recours à nos colonnes pour l'éclaircissement des idées et des suggestions ${ }^{64}$. " Le journal devint malgré tout en moins d'un mois «l'organe officiel» de la Moving Picture Exhibitors' Association ${ }^{65}$ - les propriétaires de cinéma mêmes avec qui les opérateurs étaient constamment à couteaux tirés - et après cette date on vit clairement le journal se démener entre sa fonction officielle et ses sympathies pour les opérateurs. En décembre 1907, il défendait la certification obligatoire et dévoilait ses penchants syndicalistes, probablement un peu trop au goût des exploitants: «[...] si tous les 5000 opérateurs connus se donnaient la main, ils exigeraient leur reconnaissance au sein du pays sous un drapeau distinct qu'il leur soit propre, et s'assureraient de l'élimination des opérateurs négligents ${ }^{66}$. "

\section{Traduit de l'anglais par Nicolas Dulac}

\section{NOTES}

1. L'auteur aimerait remercier Lauren Rabinovitz pour sa lecture de l'une des premières versions de cet article; Carey Williams pour lui avoir donné l'opportunité de voir certains des projecteurs dont il est ici question; et Kathryn MacKay pour son savoir, technique et autre. Cette recherche a été menée à l'Université d'Iowa et à la Bibliothèque nationale, ainsi qu'à la Bibliothèque de l'arsenal, la Bibliothèque du film et la bibliothèque Sainte-Geneviève à Paris. L'auteur apprécierait grandement toute information à propos des opérateurs de vues animées ou des techniques et dispositifs de projection durant la période du cinéma muet, n'importe où dans le monde (mais spécialement en dehors des pays mentionnés dans cet article), information que le lecteur croirait être d'une certaine pertinence pour la rédaction d'un livre consacré à ce sujet. La seconde partie de ce texte fera l'objet d'une parution ultérieure dans CiNéMAS. Il y sera notamment question de l'importance de l'opérateur-projectionniste eu égard à la réception spectatorielle des films ainsi qu'aux problèmes de narrativité dans le cinéma des premiers temps.

2. Moving Picture World, 26 février 1910.

3. Billboard, 15 actobre 1910.

4. Bioscope, 9 octobre 1908.

5. Moving Picture World, 26 novembre 1910.

L'opérateur de vues animées: deus ex machina des premières salles de cinéma 
6. Voir "A Lady Kinematograph Operator», Kinematograph and Lantern Weekly, 17 octobre 1907, pour la description d'une certaine "Senora Spencer", qui projetait seule les spectacles organisés par elle et son mari en Australie. Pour une satire anticipant le désir des femmes à devenir projectionnistes, voir "Grand Opening of the School for Lady Operators (We Don't Think)", Bioscope, 29 juin 1909.

7. Bioscope, 18 septembre 1908.

8. Pour le plan d'un nickelodeon typique, voir David S. Hullfish (1911, p. 177).

9. Bioscope, 17 novembre 1910 .

10. Extraits de l'Ordonnance de police concernant les théatres (pour Paris seulement) du 10 août 1908, qu'on peut trouver dans Kress (p. 96 sq). La dimension des cabines était réglementée par l'article 175 et la ventilation par l'article 177 . Pour les différentes lois d'États régissant la projection aux États-Unis, autour de la fin des années dix et le début des années vingt, voir James Cameron (1922, p. 102 sq.). Pour la législation anglaise, voir la note 47.

11. Ciné-Journal, 4 mars 1911.

12. Moving Picture World, 29 février 1908. L'auteur, Willis Elliott Reynolds, poursuit son inventaire des risques causés à la santé par les cabines de projection, tels que les hausses de pression, les insuffisances cardiaques, l'anémie, etc. En France, et ce aussi tardivement qu'en 1939, une thèse doctorale en médecine détaillait les différents effets nuisibles de la projection sur la santé: maladies de peau (dues à l'exposition aux ultraviolets, aux infra-rouges et à la poussière métallique); problèmes respiratoires (inhalation des vapeurs, de la poussière et de la fumée); empoisonnement (inhalation des vapeurs toxiques, des particules métalliques et de l'acide sulfurique); et même l'impuissance (causé par les rayons X émanant de l'arc électrique). L'auteur fait remarquer que selon la loi française de 1919 concernant la sécurité au travail, toujours en vigueur au moment où il rédigeait sa thèse, les opérateurs n'étaient pas considérés comme des individus pouvant souffrir de maladies indemnisables, puisqu'un accident était nécessaire pour entamer une réclamation. Voir Marguerite Guimpel-Levitsky (1939).

13. Moving Picture World, 29 février 1908.

14. Nickelodeon, mars 1909.

15. Nickelodeon, juin 1909.

16. Aux États-Unis, un commentateur mentionnait une augmentation de cent pour cent par année, entre 1908 et 1909 , ce qui élevait le nombre de salles de cinéma à dix mille (Film Index, 20 février 1909). Les taux d'augmentation étaient moins spectaculaires en France et en Angleterre, mais ils y restent tout de même significatifs. Voir Michael Chanan (1980, p. 258); Richard Abel (1988, p. 31-33); et Jean-Jacques Meusy (1996, p. 81-96).

17. Moving Picture World, 6 mars 1909.

18. Moving Picture World, 9 mai 1908.

19. Views and Film Index, 6 juin 1908.

20. Views and Film Index, 6 juin 1908.

21. Bioscope, 4 février 1909.

22. Bioscope, 28 octobre 1909.

23. Bioscope, 28 avril 1910.

24. Ciné-Journal, 31 décembre 1910.

25. Ciné-Journal, 21 janvier 1911.

26. Moving Picture World, 19 juin 1909.

27. Moving Picture World, 27 février 1909.

28. Moving Picture World, 28 septembre 1907. 
29. Billboard, 2 décembre 1911.

30. Billboard, 2 décembre 1911.

31. Cité dans Nickelodeon, 15 juin 1910.

32. Bioscope, 16 octobre 1908.

33. Moving Picture World, 19 septembre 1908.

34. Robert Osborne Baker, The International Alliance of Theatrical and Stage Employes [sic] and Moving Picture Machine Operators of the United States and Canada, p. 80.

35. Ciné-Journal, 18 juin 1910.

36. Views and Film Index, 25 avril 1908.

37. Ciné-Journal, 26 novembre 1908.

38. Moving Picture World, 25 juillet 1908 et 19 avril 1909.

39. Bioscope, 17 décembe 1908.

40. Billboard, 23 décembre 1911.

41. Ciné-Journal, 11 octobre 1909.

42. Ciné-Joumal, 27 septembre 1909.

43. Ciné-Journal, 11 juin 1909.

44. Le Courrier cinématographique, 2 septembre 1911.

45. Kinematograph and Lantern Weekly, Icr août 1907 et 30 janvier 1908.

46. Kinematograiph and Lantern Weekly, 6 février 1908.

47. Des extraits du règlement de 1900 et de 1906 du L.C.C., concernant le cinéma, peuvent être trouvés dans Kinematograph and Lantern Weekly, 21 janvier 1908. La réglementation nationale de 1909 est reproduite dans Bioscope, 23 décembre 1909 et dans Modern Bioscope Operator, $2^{c}$ édition, Londres, Ganes, 1911, p. 153-162. Pour de l'information sur cette ló et la réglementation du secteur de l'exhibition, voir David R. Williams (1997, p. 341-350).

48. Ciné-Journal, 31 décembre 1910.

49. Bioscope, 21 avril 1910.

50. Bioscope, 19 février 1911.

51. Film Index, 25 juin 1910.

52. Vieus and Film Index, 21 mars 1908.

53. Moving Picture World, 14 décembre 1907 et 8 février 1908; Views and Film Index, 23 novembre 1907.

54. Views and Film Index, 21 août 1909.

55. Pour un relevé de ce conflit en Angleterre, voir Michael Chanan (1976) et Rachael Low (1949, p. 70-75). Le conflit peut aussi être suivi dans la presse spécialisée Bioscope, exemplaires datant du 7 et 21 avril 1910; 9 février 1911 ; et 2, 9, 16 et 23 mars 1911. Du côté des États-Unis, voir Variety, 31 août 1907; et Robert Osborne Baker, The International Alliance of Theatrical and Stage Employes [sic], passim.

56. Kinematograph and Lantern Weekly, 16 mai 1907.

57. Bioscope, 17 janvier 1909.

58. Bioscope, 9 septembre 1909.

59. Modern Bioscope Operator, Londres, Ganes, 1910 ( $1^{\text {rc }}$ édition) et 1911 (2 $2^{c}$ édition).

60. Bioscope, 28 avril 1910.

61. Ciné-Journal, 6 octobre 1908.

62. Courrier cinématographique, 11 août 1911.

L'opérateur de vues animées: deus ex machina des premières salles de cinéma 
63. Moving Picture World, 9 mars 1907.

64. Moving Picture World, 18 mai 1907.

65. Moving Picture World, 29 juin 1907.

66. Moving Picture World, 14 décembre 1907.

\section{RÉFÉRENCES BIBLIOGRAPHIQUES}

Abel, 1988: Richard Abel, The Ciné Goes to Town: French Cinema 1896-1914, édition revisée, Berkeley, University of California Press, 1988.

Baker, 1933 : Robert Osborne Baker, The International Alliance of Theatrical and Stage Employes [sic] and Moving Picture Machine Operators of the United States and Canada, Lawrence, Kansas, 1933.

Cameron, 1922: James Cameron, Motion Picture Projection, $3^{\text {e }}$ édition, New York, Technical Book Co., 1922.

Chanan, 1976: Michael Chanan, Labour Power in the British Film Industry, Londres, British Film Institute, 1976.

Chanan, 1980: Michael Chanan, The Dream that Kicks: The Prehistory and Early Years of Cinema in Britain, Londres, Routledge and Kegan Paul, 1980.

Ducom, 1924 : Jacques Ducom, Le Cinématographe scientifique et industriel. Son évolution intellectuelle, sa puissance éducative et morale. Traité pratique de cinématographie, $2^{e}$ édition, Paris, Albin Michel, 1924.

Guimpel-Levitzky, 1939: Marguerite Guimpel-Levitzky, Etude sur les maladies professionnelles des opérateurs-projectionnistes du cinéma, Paris, Vigot Frères, 1939.

Hullfish, 1911: David S. Hullfish, Cyclopedia of Motion Picture Work, vol. 2, Chicago, American School of Correspondance, 1911.

Kress: E. Kress, Cathéchisme de l'opérateur de cinéma. Réponse aux questions du certificat d'aptitudes professionnelles des opérateurs projectionnistes du cinématographe, Paris, Charles-Mendel, sans date.

Le Fraper, [1912]: Charles Le Fraper, Les Projections animées. Manuel pratique à l'usage des directeurs de cinéma, des opérateurs et de toutes les personnes sintéressant à la cinématographie, Paris, Courrier cinématographique, [1912].

Low, 1949: Rachael Low, The History of British Film, 1906-1914, Londres, George Allen and Unwin, 1949.

Modern Bioscope Operator, $2^{c}$ édition, Londres, Ganes, 1911.

Meusy, 1996: Jean-Jacques Meusy, "Palaces and Holes in the Wall: Conditions of Exhibition in Paris on the Eve of World War I ", The Velvet Light Trap, n 37, 1996, p. 81-96.

Musser, 1990 : Charles Musser, The Emergence of Cinema: The American Screen to 1907, Berkeley, University of California Press, 1990.

Richardson, 1910 : F. H. Richardson, Motion Picture Handbook. A Guide for Managers and Operators of Motion Picture Theatres, $1^{\text {re }}$ édition, New York, Moving Picture World, 1910.

Rosenblatt, 1998: Nina Lara Rosenblatt, "Photogenic Neurasthenia: On Mass and Medium in the 1920's", October, n'86, 1998, p. $47-49$.

Ross, 1941 : Murray Ross, Stars and Strikes: Unionization of Hollywood, New York, Columbia University Press, 1941

Williams, 1997: David R. Williams, "The Cinematograph Act of 1909: An Introduction to the Impetus Behind the Legislation and Some Early Effects ", Film History, n 9,1997 , p. 341-350. 